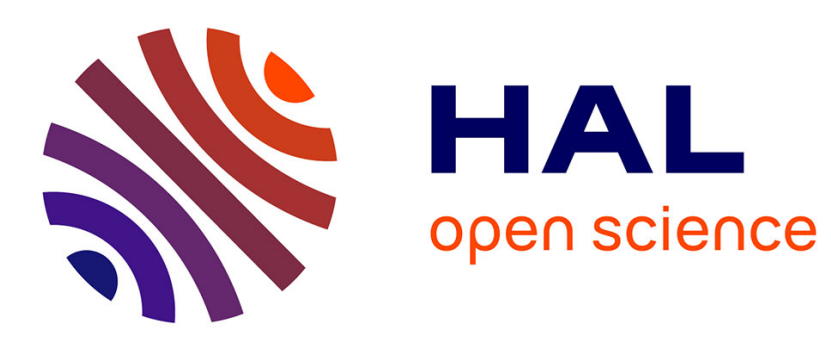

\title{
An error estimate for the Signorini problem with Coulomb friction approximated by finite elements

\author{
Patrick Hild, Yves Renard
}

\section{To cite this version:}

Patrick Hild, Yves Renard. An error estimate for the Signorini problem with Coulomb friction approximated by finite elements. SIAM Journal on Numerical Analysis, 2007, 45 (5), pp.2012-2031. 10.1137/050645439. hal-00690597

\section{HAL Id: hal-00690597 \\ https://hal.science/hal-00690597}

Submitted on 21 Apr 2018

HAL is a multi-disciplinary open access archive for the deposit and dissemination of scientific research documents, whether they are published or not. The documents may come from teaching and research institutions in France or abroad, or from public or private research centers.
L'archive ouverte pluridisciplinaire HAL, est destinée au dépôt et à la diffusion de documents scientifiques de niveau recherche, publiés ou non, émanant des établissements d'enseignement et de recherche français ou étrangers, des laboratoires publics ou privés. 


\title{
AN ERROR ESTIMATE FOR THE SIGNORINI PROBLEM WITH COULOMB FRICTION APPROXIMATED BY FINITE ELEMENTS
}

\author{
PATRICK HILD ${ }^{\dagger}$ AND YVES RENARD $\ddagger$
}

\begin{abstract}
The present paper is concerned with the unilateral contact model and the Coulomb friction law in linear elastostatics. We consider a mixed formulation in which the unknowns are the displacement field and the normal and tangential constraints on the contact area. The chosen finite element method involves continuous elements of degree one and continuous piecewise affine multipliers on the contact zone. A convenient discrete contact and friction condition is introduced in order to perform a convergence study. We finally obtain a first a priori error estimate under the assumptions ensuring the uniqueness of the solution to the continuous problem.
\end{abstract}

Key words. unilateral contact, Coulomb friction, uniqueness of solution, finite elements, a priori error estimate

Introduction. This study deals with the unilateral contact problem governed by the Coulomb friction law in linear elasticity. We consider a simplified model, the socalled static friction problem, which roughly corresponds to an incremental problem in the time discretized quasi-static model and whose solutions are also some particular equilibrium configurations of the dynamic problem.

From a mathematical point of view the early progress made on the static problem was accomplished in $[15,17]$. These studies concerned the weak formulation of the problem. The first existence results were obtained in [39] for an infinite elastic strip. Thereafter, many existence results followed for general domains, in particular in [18] (see also the references quoted therein). These existence results hold for small friction coefficients, and uniqueness is not discussed. In fact, uniqueness does not hold in the general case, at least for large friction coefficients; see [26, 27]. More recently a first uniqueness result has been obtained in [41] with the assumption that a "regular" solution exists and that the friction coefficient is sufficiently small. Additionally, the so-called nonlocal Coulomb frictional models mollifying the normal stresses were introduced in [16] and developed in [14, 11,31]. The smoothing map used in the nonlocal friction model allows one to obtain existence results for any friction coefficient. Moreover, uniqueness of a solution can also be established if the friction coefficient is small enough (see $[16,14,11,31]$ ). The same type of result (existence for any friction coefficient and uniqueness for small friction coefficients) was obtained in [32, 33] for the normal compliance model, introduced in [40,37].

From a numerical point of view, the finite element method is commonly used when approximating such frictional contact problems (see, e.g., [31, 24, 21, 35, 43]). It is well known that the finite element problem, associated with the continuous static

\footnotetext{
${ }^{\dagger}$ Laboratoire de Mathématiques de Besançon, CNRS UMR 6623, Université de Franche-Comté, 16 route de Gray, 25030 Besançon, France (patrick.hild@univ-fcomte.fr).

${ }^{\ddagger}$ MIP, CNRS UMR 5640, INSAT, Complexe scientifique de Rangueil, 31077 Toulouse, France (yves.renard@insa-lyon.fr)
} 
Coulomb friction model, always admits a solution and that the solution is unique if the friction coefficient is small enough. (Unfortunately the definition of small depends on the discretization parameter, and the bound ensuring uniqueness vanishes as the mesh is refined; see, e.g., [24].) The former result holds for any reasonable choice of the approximated contact and friction conditions (see [30]). Moreover, a first convergence study of the finite element problem towards the continuous model was accomplished in [22], where convergence was obtained under the assumptions ensuring the existence of a solution in [39] (i.e., small friction coefficient). This result proves the existence of a subsequence of discrete solutions converging towards a solution to the continuous problem. A similar result is obtained in [42] for the quasi-static model.

Our purpose is to carry out a convergence analysis and to obtain an a priori error estimate for a finite element discretization of the frictional contact conditions under the assumptions ensuring the uniqueness of a solution to the continuous problem obtained in [41]. As far as we know, this work presents the first error estimate with a convergence rate for this model.

Our paper is outlined as follows. Section 1 is concerned with the setting of the continuous problem, several equivalent weak formulations, and a presentation of the tools and techniques leading to the uniqueness result. In section 2 we consider a discretization of the problem with finite elements of degree one and continuous piecewise affine multipliers on the contact zone. We introduce a convenient discrete contact and friction condition which allows us to perform a convergence analysis and to obtain an a priori estimate of the discretization error with a quasi-optimal convergence rate of order $h^{1 / 2}$ in the energy norm under $H^{(3 / 2)+\varepsilon}$-regularity assumptions on the displacements.

1. The Signorini problem with Coulomb friction. Let $\Omega \subset R^{d}(d=2$ or 3 ) be a polygonal domain representing the reference configuration of a linearly elastic body whose boundary $\partial \Omega$ consists of three nonoverlapping open parts $\Gamma_{N}, \Gamma_{D}$, and $\Gamma_{C}$ with $\overline{\Gamma_{N}} \cup \overline{\Gamma_{D}} \cup \overline{\Gamma_{C}}=\partial \Omega$. We assume that the measures of $\Gamma_{C}$ and $\Gamma_{D}$ are positive and, in order to simplify that $\Gamma_{C}$ is a straight line segment when $d=2$ or a plane surface when $d=3$. The body is submitted to a Neumann condition on $\Gamma_{N}$ with a density of loads $F \in\left(L^{2}\left(\Gamma_{N}\right)\right)^{d}$, a Dirichlet condition on $\Gamma_{D}$ (the body is assumed to be clamped on $\Gamma_{D}$ to simplify), and to volume loads denoted $f \in\left(L^{2}(\Omega)\right)^{d}$ in $\Omega$. Finally, a unilateral contact condition with static Coulomb friction between the body and a flat rigid foundation holds on $\Gamma_{C}$ (see Figure 1.1).

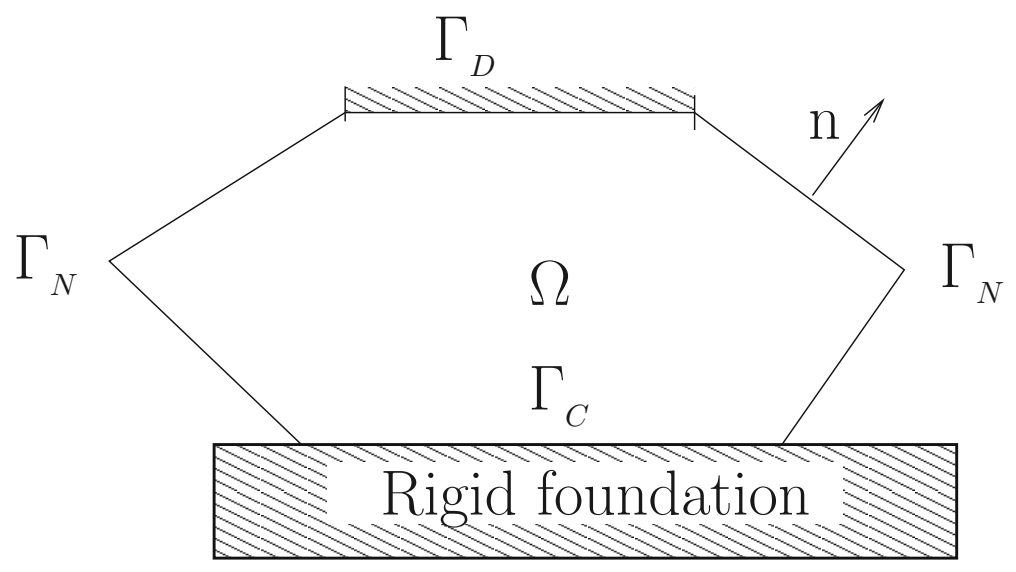

FIG. 1.1. Elastic body $\Omega$ in frictional contact. 
The problem consists of finding the displacement field $u: \bar{\Omega} \rightarrow R^{d}$ satisfying

$$
\begin{array}{rc}
-\operatorname{div} \sigma(u)=f & \text { in } \Omega, \\
\sigma(u)=\mathcal{A} \varepsilon(u) & \text { in } \Omega, \\
\sigma(u) \mathrm{n}=F & \text { on } \Gamma_{N}, \\
u=0 & \text { on } \Gamma_{D},
\end{array}
$$

where $\sigma(u)$ represents the stress tensor field, $\varepsilon(u)=\left(\nabla u+(\nabla u)^{T}\right) / 2$ denotes the linearized strain tensor field, n stands for the outward unit normal to $\Omega$ on $\partial \Omega$, and $\mathcal{A}$ is the fourth order elastic coefficient tensor which satisfies the usual symmetry and ellipticity conditions and whose components are in $L^{\infty}(\Omega)$.

On $\Gamma_{C}$, we decompose the displacement and the stress vector fields in normal and tangential components as follows:

$$
\begin{gathered}
u_{N}=u \cdot \mathrm{n}, \quad u_{T}=u-u_{N} \mathrm{n}, \\
\sigma_{N}(u)=(\sigma(u) \mathrm{n}) \cdot \mathrm{n}, \quad \sigma_{T}(u)=\sigma(u) \mathrm{n}-\sigma_{N}(u) \mathrm{n} .
\end{gathered}
$$

The unilateral contact condition on $\Gamma_{C}$ is expressed by the following complementary condition:

$$
u_{N} \leq 0, \quad \sigma_{N}(u) \leq 0, \quad u_{N} \sigma_{N}(u)=0,
$$

where a vanishing gap between the elastic solid and the rigid foundation has been chosen in the reference configuration.

Denoting by $\mathcal{F} \geq 0$ the given friction coefficient on $\Gamma_{C}$ (which is supposed constant for the sake of simplicity), the static Coulomb friction condition reads as

$$
\begin{aligned}
& \text { if } u_{T}=0 \text {, then }\left|\sigma_{T}(u)\right| \leq-\mathcal{F} \sigma_{N}(u), \\
& \text { if } u_{T} \neq 0 \text {, then } \sigma_{T}(u)=\mathcal{F} \sigma_{N}(u) \frac{u_{T}}{\left|u_{T}\right|} .
\end{aligned}
$$

When $\mathcal{F}=0$ the friction conditions (1.6)-(1.7) merely reduce to $\sigma_{T}(u)=0$ on $\Gamma_{C}$.

1.1. Classical weak formulations. This section is devoted to the presentation of different and equivalent weak formulations of the Coulomb friction problem. Let us introduce the following Hilbert spaces:

$$
\begin{gathered}
V=\left\{v \in\left(H^{1}(\Omega)\right)^{d}, v=0 \text { on } \Gamma_{D}\right\}, \\
X=\left\{\left.v\right|_{\Gamma_{C}}: v \in V\right\} \subset\left(H^{1 / 2}\left(\Gamma_{C}\right)\right)^{d}, \\
X_{N}=\left\{\left.v_{N}\right|_{\Gamma_{C}}: v \in V\right\}, \quad X_{T}=\left\{\left.v_{T}\right|_{\Gamma_{C}}: v \in V\right\},
\end{gathered}
$$

and their topological dual spaces $V^{\prime}, X^{\prime}, X_{N}^{\prime}$, and $X_{T}^{\prime}$, endowed with their usual norms. Since $\Gamma_{C}$ is a straight line segment $(d=2)$ or a plane surface $(d=3)$, we have $H_{\mathrm{oo}}^{1 / 2}\left(\Gamma_{C}\right) \subset X_{N} \subset H^{1 / 2}\left(\Gamma_{C}\right),\left(H_{00}^{1 / 2}\left(\Gamma_{C}\right)\right)^{d-1}, \subset X_{T} \subset\left(H^{1 / 2}\left(\Gamma_{C}\right)\right)^{d-1}$, which implies $X_{N}^{\prime} \subset H^{-1 / 2}\left(\Gamma_{C}\right)$ and $X_{T}^{\prime} \subset\left(H^{-1 / 2}\left(\Gamma_{C}\right)\right)^{d-1}$, where we denote by $H^{s}$ the standard Sobolev spaces (see [1]). Classically, $H^{1 / 2}\left(\Gamma_{C}\right)$ is the space of the restrictions on $\Gamma_{C}$ 
of traces on $\partial \Omega$ of functions in $H^{1}(\Omega)$, and $H^{-1 / 2}\left(\Gamma_{C}\right)$ is the dual space of $H_{00}^{1 / 2}\left(\Gamma_{C}\right)$, which is the space of the restrictions on $\Gamma_{C}$ of functions in $H^{1 / 2}(\partial \Omega)$ vanishing outside $\Gamma_{C}$. We refer to $[36,1,31]$ for a detailed presentation of trace operators and/or trace spaces.

The set of admissible displacements satisfying the noninterpenetration conditions on the contact zone is

$$
K=\left\{v \in V, v_{N} \leq 0 \quad \text { a.e. on } \Gamma_{C}\right\} .
$$

Take as given the following forms for any $u$ and $v$ in $V$ :

$$
\begin{gathered}
a(u, v)=\int_{\Omega} \mathcal{A} \varepsilon(u): \varepsilon(v) d \Omega, \\
l(v)=\int_{\Omega} f \cdot v d \Omega+\int_{\Gamma_{N}} F \cdot v d \Gamma,
\end{gathered}
$$

which represent the virtual work of the elastic forces and of the external loads, respectively. If $\langle\cdot, \cdot\rangle_{X_{N}^{\prime}, X_{N}}$ stands for the duality pairing between $X_{N}^{\prime}$ and $X_{N}$, then the "virtual work" of the friction forces is given by

$$
j\left(\mathcal{F} \lambda_{N}, v_{T}\right)=-\left\langle\mathcal{F} \lambda_{N},\left|v_{T}\right|\right\rangle_{X_{N}^{\prime}, X_{N}}
$$

for any $\lambda_{N} \in X_{N}^{\prime}$ and $v_{T} \in X_{T}$. From the previous assumptions it follows that

$a(\cdot, \cdot)$ is a bilinear symmetric $V$-elliptic and continuous form on $V \times V$ :

$\exists \alpha>0, \exists M>0, \quad a(v, v) \geq \alpha\|v\|_{V}^{2}, a(u, v) \leq M\|u\|_{V}\|v\|_{V} \quad \forall u, v \in V$,

$l(\cdot)$ linear continuous form on $V, \quad$ i.e., $\exists L>0, \quad|l(v)| \leq L\|v\|_{V} \quad \forall v \in V$.

Moreover, $j\left(\mathcal{F} \lambda_{N}, v_{T}\right)$ is linear continuous with respect to $\lambda_{N}$ and convex lower semicontinuous with regard to $v_{T}$ if $\lambda_{N}$ is a nonpositive element of $X_{N}^{\prime}$ (see, for instance, $[2])$.

Clearly $a(\cdot, \cdot)$ is an inner product on $V$, and the associated norm,

$$
\|v\|_{a}=(a(v, v))^{1 / 2}
$$

is equivalent to the usual norm of $V$ :

$$
\sqrt{\alpha}\|v\|_{V} \leq\|v\|_{a} \leq \sqrt{M}\|v\|_{V} \quad \forall v \in V .
$$

The continuity constant of $l(\cdot)$ can also be given with respect to $\|\cdot\|_{a}$ :

$$
\exists L_{a}>0, \quad|l(v)| \leq L_{a}\|v\|_{a} \quad \forall v \in V .
$$

Constants $L$ and $L_{a}$ can be chosen such that

$$
\sqrt{\alpha} L_{a} \leq L \leq \sqrt{M} L_{a}
$$

The weak formulation of problem (1.1)-(1.7) (written as an inequality), introduced in $[15]$ (see also [17]), is

$$
\left\{\begin{array}{l}
\text { Find } u \in K \text { satisfying } \\
a(u, v-u)+j\left(\mathcal{F} \sigma_{N}(u), v_{T}\right)-j\left(\mathcal{F} \sigma_{N}(u), u_{T}\right) \geq l(v-u) \quad \forall v \in K .
\end{array}\right.
$$


Introducing the stresses on the contact boundary as an unknown in the previous formulation, one obtains the following equivalent formulation (see [30]):

$$
\left\{\begin{array}{l}
\text { Find } u \in V, \lambda_{N} \in X_{N}, \text { and } \lambda_{T} \in X_{T} \text { satisfying } \\
a(u, v)=l(v)+\left\langle\lambda_{N}, v_{N}\right\rangle_{X_{N}^{\prime}, X_{N}}+\left\langle\lambda_{T}, v_{T}\right\rangle_{X_{T}^{\prime}, X_{T}} \quad \forall v \in V, \\
u_{N} \leq 0,\left\langle\lambda_{N}, v_{N}-u_{N}\right\rangle_{X_{N}^{\prime}, X_{N}} \geq 0 \quad \forall v_{N} \in X_{N}, v_{N} \leq 0, \\
\left\langle\lambda_{T}, v_{T}-u_{T}\right\rangle_{X_{T}^{\prime}, X_{T}}+j\left(\mathcal{F} \lambda_{N}, v_{T}\right)-j\left(\mathcal{F} \lambda_{N}, u_{T}\right) \geq 0 \quad \forall v_{T} \in X_{T} .
\end{array}\right.
$$

Inverting contact and friction relations, one also obtains the classical equivalent hybrid formulation (see [30]):

$$
\left\{\begin{array}{l}
\text { Find } u \in V, \lambda_{N} \in X_{N}, \text { and } \lambda_{T} \in X_{T} \text { satisfying } \\
a(u, v)=l(v)+\left\langle\lambda_{N}, v_{N}\right\rangle_{X_{N}^{\prime}, X_{N}}+\left\langle\lambda_{T}, v_{T}\right\rangle_{X_{T}^{\prime}, x_{T}} \quad \forall v \in V, \\
\lambda_{N} \in \Lambda_{N}, \quad\left\langle\mu_{N}-\lambda_{N}, u_{N}\right\rangle_{X_{N}^{\prime}, X_{N}} \geq 0 \quad \forall \mu_{N} \in \Lambda_{N}, \\
\lambda_{T} \in \Lambda_{T}\left(\mathcal{F} \lambda_{N}\right),\left\langle\mu_{T}-\lambda_{T}, u_{T}\right\rangle_{X_{T}^{\prime}, X_{T}} \geq 0 \quad \forall \mu_{T} \in \Lambda_{T}\left(\mathcal{F} \lambda_{N}\right),
\end{array}\right.
$$

where $\Lambda_{N}$ and $\Lambda_{T}\left(\mathcal{F} \lambda_{N}\right)$ denote the sets of admissible normal and tangential stresses:

$$
\begin{gathered}
\Lambda_{N}=\left\{\lambda_{N} \in X_{N}^{\prime}:\left\langle\lambda_{N}, v_{N}\right\rangle_{X_{N}^{\prime}, X_{N}} \geq 0 \quad \forall v_{N} \leq 0\right\}, \\
\Lambda_{T}\left(\mathcal{F} \lambda_{N}\right)=\left\{\lambda_{T} \in X_{T}^{\prime}:\left\langle\lambda_{T}, v_{T}\right\rangle_{X_{T}^{\prime}, X_{T}}+j\left(\mathcal{F} \lambda_{N}, v_{T}\right) \geq 0, \quad \forall v_{T} \in X_{T}\right\} .
\end{gathered}
$$

It is easy to check that the multipliers $\lambda_{N}$ and $\lambda_{T}$ solving (1.9) and (1.10) satisfy $\lambda_{N}=\sigma_{N}(u)$ and $\lambda_{T}=\sigma_{T}(u)$ at least in a weak sense. The main difficulty in the existence and uniqueness analysis of (1.8), (1.9), or (1.10) comes from the coupling between the friction threshold $\mathcal{F} \sigma_{N}(u)$ and the contact pressure $\sigma_{N}(u)$.

Remark 1. The equivalence between problems (1.8) and (1.9) is easy to obtain here since the assumption $f \in L^{2}(\Omega)^{d}$ implies that a generalized Green formula holds (see [31], for instance). The proof can also be made directly as follows. A solution to problem (1.9) is obviously a solution to problem (1.8). Conversely, if $u$ is solution to problem (1.8), then the map $X \ni v \longmapsto a(u, \Pi(v))-l(\Pi(v))$ is linear continuous for any continuous lifting operator $\Pi: X \rightarrow V$. Thus there exists $\lambda \in X^{\prime}$ such that $\langle\lambda, v\rangle_{X^{\prime}, X}=a(u, \Pi(v))-l(\Pi(v))$ for all $v \in X$. It is easy to state that in fact $\langle\lambda, v\rangle_{X^{\prime}, X}=a(u, v)-l(v)$ for all $v \in V$, proving $a\left(u, \Pi\left(\left.v\right|_{\Gamma_{C}}\right)-v\right)-l\left(\Pi\left(\left.v\right|_{\Gamma_{C}}\right)-v\right)=0$ for all $v \in V$. Indeed, $\Pi\left(\left.v\right|_{\Gamma_{C}}\right)-v$ has a vanishing trace on $\Gamma_{C}$, and replacing successively $v-u$ by $\left(\Pi\left(\left.v\right|_{\Gamma_{C}}\right)-v+u\right)-u$ and by $\left(v-\Pi\left(\left.v\right|_{\Gamma_{C}}\right)+u\right)-u$ in the inequality of (1.8) leads to this result. The two inequalities of (1.9) result then from the replacement of $a(u, v-u)-l(v-u)$ by $\langle\lambda, v-u\rangle_{X^{\prime}, X}$ in the inequality of (1.8), separating normal and tangential components $\left(\Gamma_{C}\right.$ is straight here), and remarking that applying a Green formula, one has $\sigma_{N}(u)=\lambda_{N}$. The equivalence between (1.9) and (1.10) is developed in [30] by computing the Fenchel conjugate of $j\left(\mathcal{F} \sigma_{N}, \cdot\right)$ and inverting the normal cone to $K$. 
1.2. Neumann to Dirichlet operator. We introduce the Neumann to Dirichlet operator on $\Gamma_{C}$ and its basic properties. This will allow us to restrict the contact and friction problem on $\Gamma_{C}$ and obtain useful estimates.

Let $\lambda=\left(\lambda_{N}, \lambda_{T}\right) \in X^{\prime}$. The solution $u$ to

$$
\left\{\begin{array}{l}
\text { Find } u \in V \text { satisfying } \\
a(u, v)=l(v)+\langle\lambda, v\rangle_{X^{\prime}, X}
\end{array} \quad \forall v \in V\right.
$$

is unique (see [17]). So it is possible to define the operator

$$
\begin{aligned}
\mathbb{E}: X^{\prime} & \longrightarrow X \\
\lambda & \left.\longmapsto\right|_{\Gamma_{\Gamma_{C}}}
\end{aligned}
$$

It is easy to check that the operator $\mathbb{E}$ is affine and continuous. We define the following norms on $\Gamma_{C}$ relative to $a(\cdot, \cdot)$ :

$$
\begin{gathered}
\|v\|_{a, \Gamma_{C}}=\inf _{w \in V,\left.w\right|_{\Gamma_{C}}=v}\|w\|_{a}, \\
\left\|v_{N}\right\|_{a, \Gamma_{C}}=\inf _{\substack{w \in V \\
w_{N}=v_{N} \text { on } \Gamma_{C}}}\|w\|_{a}=\inf _{\substack{w \in V, w_{N}=v_{N} \Gamma_{C}}}\|w\|_{a, \Gamma_{C}}, \\
\left\|v_{T}\right\|_{a, \Gamma_{C}}=\inf _{\substack{w \in V, w_{T}=v_{T} \text { on } \Gamma_{C}}}\|w\|_{a}=\inf _{\substack{w \in V, w_{T}=v_{T} \text { on } \Gamma_{C}}}\|w\|_{a, \Gamma_{C}}, \\
\|\lambda\|_{-a, \Gamma_{C}}=\sup _{\substack{v \in X \\
v \neq 0}} \frac{\langle\lambda, v\rangle_{X^{\prime}, X}}{\|v\|_{a, \Gamma_{C}}}=\sup _{\substack{v \in V \\
v \neq 0}} \frac{\langle\lambda, v\rangle_{X^{\prime}, X}}{\|v\|_{a}}, \\
\left\|\lambda_{N}\right\|_{-a, \Gamma_{C}}=\sup _{\substack{v_{N} \in X_{N} \\
v_{N} \neq 0}} \frac{\left\langle\lambda_{N}, v_{N}\right\rangle_{X_{N}^{\prime}, X_{N}}}{\left\|v_{N}\right\|_{a, \Gamma_{C}}}=\left\|\left(\lambda_{N}, 0\right)\right\|_{-a, \Gamma_{C}}, \\
\left\|\lambda_{T}\right\|\left\|_{-a, \Gamma_{C}}=\sup _{\substack{v_{T} \in X_{T} \\
v_{T} \neq 0}} \frac{\left\langle\lambda_{T}, v_{T}\right\rangle_{X_{T}^{\prime}, X_{T}}}{\left\|v_{T}\right\|_{a, \Gamma_{C}}}=\right\|\left(0, \lambda_{T}\right) \|_{-a, \Gamma_{C}},
\end{gathered}
$$

which are equivalent, respectively, to the norms in $X$ and $X^{\prime}$ :

$$
\begin{gathered}
\frac{\sqrt{\alpha}}{C_{1}}\|v\|_{X} \leq\|v\|_{a, \Gamma_{C}} \leq \sqrt{M} \gamma\|v\|_{X}, \\
\frac{\sqrt{\alpha}}{C_{1}}\left\|v_{N}\right\|_{X_{N}} \leq\left\|v_{N}\right\|_{a, \Gamma_{C}} \leq \sqrt{M} \gamma\left\|v_{N}\right\|_{X_{N}}, \\
\frac{\sqrt{\alpha}}{C_{1}}\left\|v_{T}\right\|_{X_{T}} \leq\left\|v_{T}\right\|_{a, \Gamma_{C}} \leq \sqrt{M} \gamma\left\|v_{T}\right\|_{X_{T}},
\end{gathered}
$$




$$
\frac{1}{\sqrt{M} \gamma}\|\lambda\|_{X^{\prime}} \leq\|\lambda\|_{-a, \Gamma_{C}} \leq \frac{C_{1}}{\sqrt{\alpha}}\|\lambda\|_{X^{\prime}}
$$

One also has

$$
\left\|v_{N}\right\|_{a, \Gamma_{C}} \leq\|v\|_{a, \Gamma_{C}}
$$

and

$$
\left\|\lambda_{N}\right\|_{-a, \Gamma_{C}} \leq C_{\alpha}\|\lambda\|_{-a, \Gamma_{C}}
$$

with a constant $C_{\alpha} \leq C_{1} \gamma \sqrt{M / \alpha}$. (But a better estimate should be possible: following [19], $C_{\alpha}$ is close to 1 when the Poisson ratio is close to 0.)

With the previous norms, it is possible to state (see [41]) the following equalities, when $u=\mathbb{E}(\lambda)$ and $\bar{u}=\mathbb{E}(\bar{\lambda})$ are the solutions to problem (1.11):

$$
\|u-\bar{u}\|_{a}=\|\mathbb{E}(\lambda)-\mathbb{E}(\bar{\lambda})\|_{a, \Gamma_{C}}=\|\lambda-\bar{\lambda}\|_{-a, \Gamma_{C}} .
$$

1.3. Direct weak inclusion formulation. Let

$$
K_{N}=\left\{v_{N} \in X_{N}: v_{N} \leq 0 \text { a.e. on } \Gamma_{C}\right\}
$$

be the set of admissible normal displacements on $\Gamma_{C}$. The normal cone in $X_{N}^{\prime}$ to $K_{N}$ at $v_{N} \in X_{N}$ is defined as

$$
N_{K_{N}}\left(v_{N}\right)=\left\{\mu_{N} \in X_{N}^{\prime}:\left\langle\mu_{N}, w_{N}-v_{N}\right\rangle_{X_{N}^{\prime}, X_{N}} \leq 0 \forall w_{N} \in K_{N}\right\} \quad \text { if } v_{N} \in K_{N},
$$

and $N_{K_{N}}\left(v_{N}\right)=\emptyset$ if $v_{N} \notin K_{N}$. The subdifferential of $j\left(\mathcal{F} \lambda_{N},.\right)$ (i.e., with respect to the second variable) at $u_{T}$ is given by

$$
\begin{aligned}
& \partial_{2} j\left(\mathcal{F} \lambda_{N}, u_{T}\right) \\
& =\left\{\mu_{T} \in X_{T}^{\prime}: j\left(\mathcal{F} \lambda_{N}, v_{T}\right) \geq j\left(\mathcal{F} \lambda_{N}, u_{T}\right)+\left\langle\mu_{T}, v_{T}-u_{T}\right\rangle_{X_{T}^{\prime}, x_{T}} \quad \forall v_{T} \in X_{T}\right\} .
\end{aligned}
$$

With this notation, problem (1.8) can be written

$$
\left\{\begin{array}{l}
\text { Find } u \in V, \lambda_{N} \in X_{N}^{\prime}, \text { and } \lambda_{T} \in X_{T}^{\prime} \text { satisfying } \\
\left(u_{N}, u_{T}\right)=\mathbb{E}\left(\lambda_{N}, \lambda_{T}\right) \\
-\lambda_{N} \in N_{K_{N}}\left(u_{N}\right) \text { in } X_{N}^{\prime} \\
-\lambda_{T} \in \partial_{2} j\left(\mathcal{F} \lambda_{N}, u_{T}\right) \text { in } X_{T}^{\prime}
\end{array}\right.
$$

More details resulting from this equivalence can be found in [34].

1.4. A uniqueness criterion. In [26, 27] some multisolutions of the problem (1.1)-(1.7) are exhibited for triangular or quadrangular domains. These multiple solutions involve either an infinite set of slipping solutions or two isolated (stick and separation) configurations. Note that these examples of nonuniqueness involve large friction coefficients (i.e., $\mathcal{F}>1$ ) and tangential displacements with a constant sign on $\Gamma_{C}$. Actually, it seems that no multisolution has been detected for an arbitrary 
small friction coefficient in the continuous case, although such a result exists for finite element approximations in [25], but for a variable geometry. The forthcoming partial uniqueness result is obtained in [41]: it defines some cases where it is possible to affirm that a solution to the Coulomb friction problem is in fact the unique solution. More precisely, if a regular solution to the Coulomb friction problem exists (here the term regular means, roughly speaking, that the transition is smooth when the slip direction changes) and if the friction coefficient is small enough, then this solution is the only one. We recall the main useful tools leading to that result.

Lemma 1.1. Let $u$ and $\bar{u}$ be two solutions to problem (1.8), and let $\lambda$ and $\bar{\lambda}$ be the corresponding contact stresses on $\Gamma_{C}$. Then the following estimate holds:

$$
\|u-\bar{u}\|_{a}^{2}=\|\lambda-\bar{\lambda}\|_{-a, \Gamma_{C}}^{2} \leq\left\langle\zeta-\lambda_{T}, \bar{u}_{T}-u_{T}\right\rangle_{X_{T}^{\prime}, x_{T}} \quad \forall \zeta \in-\partial_{2} j\left(\mathcal{F} \bar{\lambda}_{N}, u_{T}\right) .
$$

Proof. From (1.12), the Green formula, and (1.5), we get

$$
\begin{aligned}
\|u-\bar{u}\|_{a}^{2}=\|\lambda-\bar{\lambda}\|_{-a, \Gamma_{C}}^{2} & =\left\langle\bar{\lambda}_{N}-\lambda_{N}, \bar{u}_{N}-u_{N}\right\rangle_{x_{N}^{\prime}, x_{N}}+\left\langle\bar{\lambda}_{T}-\lambda_{T}, \bar{u}_{T}-u_{T}\right\rangle_{X_{T}^{\prime}, x_{T}} \\
& \leq\left\langle\bar{\lambda}_{T}-\lambda_{T}, \bar{u}_{T}-u_{T}\right\rangle_{x_{T}^{\prime}, x_{T}} .
\end{aligned}
$$

Thus

$$
\|u-\bar{u}\|_{a}^{2} \leq\left\langle\left(\bar{\lambda}_{T}-\zeta\right)+\left(\zeta-\lambda_{T}\right), \bar{u}_{T}-u_{T}\right\rangle_{X_{T}^{\prime}, X_{T}} \quad \forall \zeta \in-\partial_{2} j\left(\mathcal{F} \bar{\lambda}_{N}, u_{T}\right) .
$$

The conclusion follows from (1.13) and the fact that $-\partial_{2} j\left(\mathcal{F} \bar{\lambda}_{N},.\right)$ is a monotone set-valued mapping.

We now introduce the space of multipliers $M\left(X_{T} \rightarrow X_{N}\right)$ of the functions $\xi$ : $\Gamma_{C} \rightarrow R^{d}$ satisfying $\xi . \mathrm{n}=0$ a.e. on $\Gamma_{C}$ and such that the following equivalent norms are finite:

$$
\|\xi\|_{M\left(X_{T} \rightarrow X_{N}\right)}=\sup _{v_{T} \in X_{T}} \frac{\left\|\xi \cdot v_{T}\right\|_{X_{N}}}{\left\|v_{T}\right\|_{X_{T}}}, \quad \text { and } \quad\|\xi\|_{a}=\sup _{\substack{v_{T} \in X_{T} \\ v_{T} \neq 0}} \frac{\left\|\xi \cdot v_{T}\right\|_{a, \Gamma_{C}}}{\left\|v_{T}\right\|_{a, \Gamma_{C}}} .
$$

Since $\Gamma_{C}$ is assumed to be straight, $M\left(X_{T} \rightarrow X_{N}\right)$ contains for any $\varepsilon>0$ the space $H^{1 / 2+\varepsilon}\left(\Gamma_{C}\right)$ when $d=2$. When $d=3, M\left(X_{T} \rightarrow X_{N}\right)$ contains $H^{1}\left(\Gamma_{C}\right) \cap L^{\infty}\left(\Gamma_{C}\right)$. (See [38] for a complete discussion on the theory of multipliers in a pair of Hilbert spaces.)

The partial uniqueness result is given assuming that $\lambda_{T}=\mathcal{F} \lambda_{N} \xi$, with $\xi \in M\left(X_{T} \rightarrow X_{N}\right)$. The product $\lambda_{N} \xi$ has to be understood in the sense that $\left\langle\lambda_{N} \xi, v_{T}\right\rangle_{X_{T}^{\prime}, X_{T}}=\left\langle\lambda_{N}, \xi \cdot v_{T}\right\rangle_{X_{N}^{\prime}, X_{N}}$ for all $v_{T} \in X_{T}$. It is easy to see that this implies $|\xi| \leq 1$ a.e. on the support of $\lambda_{N}$. More precisely, this implies that $\xi \in \operatorname{Dir}_{T}\left(u_{T}\right)$ a.e. on the support of $\lambda_{N}$, where $\operatorname{Dir}_{T}($.$) is the subdifferential of the convex map$ $R^{d} \ni x \longmapsto\left|x_{T}\right|$. This means that it is possible to assume that $\xi \in \operatorname{Dir}_{T}\left(u_{T}\right)$ a.e. on $\Gamma_{C}$.

Proposition 1.2. Let $u$ be a solution to problem (1.8) such that $\lambda_{T}=\mathcal{F} \lambda_{N} \xi$, with $\xi \in M\left(X_{T} \rightarrow X_{N}\right), \xi \in \operatorname{Dir}_{T}\left(u_{T}\right)$ a.e. on $\Gamma_{C}$, and $\mathcal{F}<\left(C_{\alpha}\|\xi\|_{a}\right)^{-1}$. Then $u$ is the unique solution to problem (1.8).

Proof. Let $\bar{u}$ be another solution to problem (1.8), where $\bar{\lambda}_{N}$ and $\bar{\lambda}_{T}$ denote the corresponding contact stresses on $\Gamma_{C}$. According to Lemma 1.1, we write

$$
\|u-\bar{u}\|_{a}^{2} \leq\left\langle\zeta-\lambda_{T}, \bar{u}_{T}-u_{T}\right\rangle_{x_{T}^{\prime}, x_{T}} \quad \forall \zeta \in-\partial_{2} j\left(\mathcal{F} \bar{\lambda}_{N}, u_{T}\right) .
$$




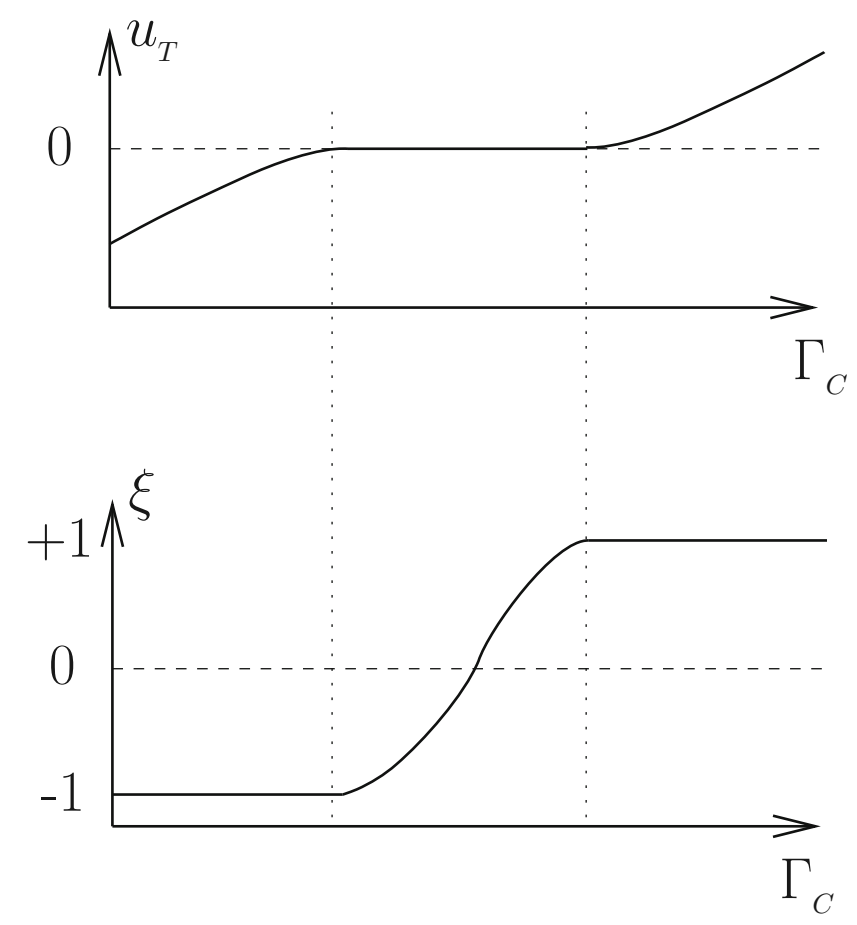

FIG. 1.2. Example of a tangential displacement $u_{T}$ and a possible corresponding multiplier $\xi$ when $d=2$.

It is easy to see that a possible choice is $\zeta=\mathcal{F} \bar{\lambda}_{N} \xi$. Therefore

$$
\begin{aligned}
\|u-\bar{u}\|_{a}^{2} \leq\left\langle\mathcal{F} \xi\left(\bar{\lambda}_{N}-\lambda_{N}\right), \bar{u}_{T}-u_{T}\right\rangle_{X_{T}^{\prime}, x_{T}} & \leq \mathcal{F}\left\|\lambda_{N}-\bar{\lambda}_{N}\right\|_{-a, \Gamma_{C}}\left\|\xi \cdot\left(u_{T}-\bar{u}_{T}\right)\right\|_{a, \Gamma_{C}} \\
& \leq C_{\alpha} \mathcal{F}\|\xi\|_{a}\|\lambda-\bar{\lambda}\|_{-a, \Gamma_{C}}\|u-\bar{u}\|_{a} \\
& =C_{\alpha} \mathcal{F}\|\xi\|_{a}\|u-\bar{u}\|_{a}^{2},
\end{aligned}
$$

which implies that $\bar{u}=u$ when $\mathcal{F}<\left(C_{\alpha}\|\xi\|_{a}\right)^{-1}$.

In two space dimensions $(d=2)$, the case $\xi \equiv 1$ corresponds to a homogeneous sliding direction, and the previous result is complementary to the nonuniqueness results obtained in $[26,27]$.

As illustrated in Figure 1.2, when $d=2$ the multiplier $\xi$ has to vary from -1 to +1 each time the sign of the tangential displacement changes from negative to positive. The set $M\left(X_{T} \rightarrow X_{N}\right)$ does not contain any multiplier having a singularity of the first kind. Consequently, in order to satisfy the assumptions of Proposition 1.2, the tangential displacement of the solution $u$ cannot pass from a negative value to a positive value and be zero at only a single point of $\Gamma_{C}$.

Remark 2. This remark deals with a more precise discussion concerning the assumption $\lambda_{T}=\mathcal{F} \lambda_{N} \xi, \xi \in M\left(X_{T} \rightarrow X_{N}\right), \xi \in \operatorname{Dir}_{T}\left(u_{T}\right)$ and the cases where the assumption cannot be fulfilled independently of the regularity of the solution when $d=2$. On the one hand, it is easy to show that the choice of $\xi$ is unique at any point where $\lambda_{N} \neq 0$ or $u_{T} \neq 0$. In the first case $\xi=\lambda_{T} /\left(\mathcal{F} \lambda_{N}\right)$, in the second case $\xi=u_{T} /\left|u_{T}\right|$, and both expressions coincide when $\lambda_{N} \neq 0$ and $u_{T} \neq 0$. On the other hand, any $\xi \in[-1,1]$ can be chosen at the points where $\lambda_{N}=u_{T}=0$. So it remains to determine when $\xi$ lies in $M\left(X_{T} \rightarrow X_{N}\right)$. If there are no points such that $\lambda_{N}=u_{T}=0$ on $\Gamma_{C}$, then the condition $\xi \in M\left(X_{T} \rightarrow X_{N}\right)$ is linked to the regularity of $u$ (in other words, if $u$ is regular enough, then $\xi \in M\left(X_{T} \rightarrow X_{N}\right)$ ). If there are some points such that $\lambda_{N}=u_{T}=0$, then it is easy to show that the continuity of $\xi$ can be lost (whatever the regularity of $u$ is) only if some of these points are isolated. A discussion 
shows then that $\xi \notin M\left(X_{T} \rightarrow X_{N}\right)$ in three cases. The first one is when $u_{T}$ passes from a negative to a positive value at such a point (note that this could also occur at a point which is separated from the foundation). The second case corresponds to a stick area surrounding such an isolated point and where the right and left limits of $\lambda_{T} /\left(\mathcal{F} \lambda_{N}\right)$ differ at this point (where $\lambda_{T}=\lambda_{N}=u_{T}=u_{N}=0$ ). The third case is a combination of both previous cases: a side where $u_{T} \neq 0$, the other one with $u_{T}=0$ and $\lambda_{N} \neq 0$, and a limit of $\lambda_{T} /\left(\mathcal{F} \lambda_{N}\right)$ which differs from $u_{T} /\left|u_{T}\right|$. If the solution is less regular, then other cases of nonfulfillment could appear, but we think that this assumption (which is needed to obtain the uniqueness of a solution to the continuous problem) takes into account many frictional contact configurations.

2. Finite element approximation. Let $V^{h} \subset V$ be a family of finite dimensional vector spaces indexed by $h$ coming from a regular family $\mathcal{T}^{h}$ (see [9]) of triangulations of the domain $\Omega$ ( $h$ represents the largest diameter among all elements). We choose standard continuous and piecewise affine functions, i.e.,

$$
V^{h}=\left\{v^{h} \in(\mathcal{C}(\bar{\Omega}))^{d},\left.v^{h}\right|_{T} \in P_{1}(T) \quad \forall T \in \mathcal{T}^{h}, v^{h}=0 \text { on } \Gamma_{D}\right\} .
$$

Define

$$
\begin{aligned}
& X_{N}^{h}=\left\{\left.v_{N}^{h}\right|_{\Gamma_{C}}: v^{h} \in V^{h}\right\}, \\
& X_{T}^{h}=\left\{\left.v_{T}^{h}\right|_{\Gamma_{C}}: v^{h} \in V^{h}\right\}, \\
& X^{h}=\left\{\left.v^{h}\right|_{\Gamma_{C}}: v^{h} \in V^{h}\right\}=X_{N}^{h} \times X_{T}^{h} .
\end{aligned}
$$

Identifying $X_{N}^{h}$ and $X_{T}^{h}$ with their dual spaces using the $L^{2}$ scalar product, we consider that $X_{N}^{h}$ and $X_{T}^{h}$ are also the finite-dimensional approximations of $X_{N}^{\prime}$ and $X_{T}^{\prime}$, respectively.

The finite element discretization of problem (1.10) becomes

$$
\left\{\begin{array}{l}
\text { Find } u^{h} \in V^{h}, \lambda_{N}^{h} \in X_{N}^{h}, \text { and } \lambda_{T}^{h} \in X_{T}^{h} \text { satisfying } \\
a\left(u^{h}, v^{h}\right)=l\left(v^{h}\right)+\int_{\Gamma_{C}} \lambda_{N}^{h} v_{N}^{h} d \Gamma+\int_{\Gamma_{C}} \lambda_{T}^{h} \cdot v_{T}^{h} d \Gamma \quad \forall v^{h} \in V^{h}, \\
\lambda_{N}^{h} \in \Lambda_{N}^{h}, \quad \int_{\Gamma_{C}}\left(\mu_{N}^{h}-\lambda_{N}^{h}\right) u_{N}^{h} d \Gamma \geq 0 \quad \forall \mu_{N}^{h} \in \Lambda_{N}^{h}, \\
\lambda_{T}^{h} \in \Lambda_{T}^{h}\left(\mathcal{F} \lambda_{N}^{h}\right), \quad \int_{\Gamma_{C}}\left(\mu_{T}^{h}-\lambda_{T}^{h}\right) \cdot u_{T}^{h} d \Gamma \geq 0 \quad \forall \mu_{T}^{h} \in \Lambda_{T}^{h}\left(\mathcal{F} \lambda_{N}^{h}\right),
\end{array}\right.
$$

where the approximations of $\Lambda_{N}$ and $\Lambda_{T}\left(\mathcal{F} \lambda_{N}\right)$ have been chosen in the following way:

$$
\Lambda_{T}^{h}\left(\mathcal{F} \lambda_{N}^{h}\right)=\left\{\lambda_{T}^{h} \in X_{T}^{h}: \int_{\Gamma_{C}} \lambda_{T}^{h} \cdot v_{T}^{h} d \Gamma+j\left(\mathcal{F} \lambda_{N}^{h}, v_{T}^{h}\right) \geq 0 \quad \forall v_{T}^{h} \in X_{T}^{h}\right\} .
$$


To simplify our discussion, we assume afterwards that the mesh inherited on the contact zone is quasi-uniform (although there exist some less restrictive assumptions; see, e.g., [13]) of size $h$ (to simplify). Another simplification is that we restrict ourselves to the two-dimensional case $(d=2)$, and we assume that the end points of $\Gamma_{C}$ do not belong to $\overline{\Gamma_{D}}$ (in other words, $\overline{\Gamma_{C}} \cap \overline{\Gamma_{D}}=\emptyset$ ). More general cases will be discussed in some remarks at the end of the paper.

With this choice of discretization, the following discrete Babuška-Brezzi inf-sup condition holds (see, e.g., $[12,6]$ ):

$$
\inf _{\lambda^{h} \in X^{h}} \sup _{v^{h} \in V^{h}} \frac{\int_{\Gamma_{C}} \lambda^{h} \cdot v^{h} d \Gamma}{\left\|v^{h}\right\|_{a}\left\|\lambda^{h}\right\|_{-a, \Gamma_{C}}} \geq c_{i s}>0,
$$

where $c_{i s} \leq 1$ is independent of $h$. As a consequence, problem (2.3) admits a solution for any friction coefficient, and the solution is unique for a sufficiently small friction coefficient (where the label "small" may depend on $h$ ) (see [30]).

The following lemma shows the relation between the hybrid formulation and the direct formulation of the friction condition in the discrete framework.

Lemma 2.1. For $\lambda_{N}^{h} \in \Lambda_{N}^{h}$, a pair $\left(\lambda_{T}^{h}, u_{T}^{h}\right) \in X_{T}^{h} \times X_{T}^{h}$ satisfies

$$
\lambda_{T}^{h} \in \Lambda_{T}^{h}\left(\mathcal{F} \lambda_{N}^{h}\right), \quad \int_{\Gamma_{C}}\left(\mu_{T}^{h}-\lambda_{T}^{h}\right) \cdot u_{T}^{h} d \Gamma \geq 0 \quad \forall \mu_{T}^{h} \in \Lambda_{T}^{h}\left(\mathcal{F} \lambda_{N}^{h}\right)
$$

if and only if the pair satisfies

$$
\int_{\Gamma_{C}} \lambda_{T}^{h} \cdot\left(v_{T}^{h}-u_{T}^{h}\right) d \Gamma+j\left(\mathcal{F} \lambda_{N}^{h}, v_{T}^{h}\right)-j\left(\mathcal{F} \lambda_{N}^{h}, u_{T}^{h}\right) \geq 0 \quad \forall v_{T}^{h} \in X_{T}^{h} .
$$

Proof. Let us first assume that $\left(\lambda_{T}^{h}, u_{T}^{h}\right)$ satisfies (2.7). For an arbitrary choice $\xi \in \mathcal{F} \lambda_{N}^{h} \operatorname{Dir}_{T}\left(u_{T}^{h}\right)$ the map $v_{T}^{h} \longmapsto \int_{\Gamma_{C}} \xi \cdot v_{T}^{h} d \Gamma$ is a linear form on $X_{T}^{h}$, and thus by the Riesz representation theorem there exists $\mu_{T}^{h} \in X_{T}^{h}$ such that $\int_{\Gamma_{C}} \mu_{T}^{h} \cdot v_{T}^{h} d \Gamma=$ $\int_{\Gamma_{C}} \xi \cdot v_{T}^{h} d \Gamma$ for all $v_{T}^{h} \in X_{T}^{h}$. This $\mu_{T}^{h}$ satisfies $\int_{\Gamma_{C}} \mu_{T}^{h} \cdot u_{T}^{h} d \Gamma=\int_{\Gamma_{C}} \mathcal{F} \lambda_{N}^{h}\left|u_{T}^{h}\right| d \Gamma=$ $-j\left(\mathcal{F} \lambda_{N}^{h}, u_{T}^{h}\right)$, and $\mu_{T}^{h}$ is an element of $\Lambda_{T}^{h}\left(\mathcal{F} \lambda_{N}^{h}\right)$. Now considering this particular $\mu_{T}^{h}$ in $(2.7)$ leads to

$$
\int_{\Gamma_{C}} \lambda_{T}^{h} \cdot u_{T}^{h} d \Gamma+j\left(\mathcal{F} \lambda_{N}^{h}, u_{T}^{h}\right) \leq 0 .
$$

Together with the fact that $\lambda_{T}^{h}$ is in $\Lambda_{T}^{h}\left(\mathcal{F} \lambda_{N}^{h}\right)$ this leads to the complementarity relation

$$
\int_{\Gamma_{C}} \lambda_{T}^{h} \cdot u_{T}^{h} d \Gamma+j\left(\mathcal{F} \lambda_{N}^{h}, u_{T}^{h}\right)=0,
$$

which straightforwardly implies (2.8).

Conversely, let us assume that $\left(\lambda_{T}^{h}, u_{T}^{h}\right)$ satisfies (2.8). Then choosing $v_{T}^{h}=$ 0 in (2.8) gives $-\int_{\Gamma_{C}} \lambda_{T}^{h} \cdot u_{T}^{h} d \Gamma-j\left(\mathcal{F} \lambda_{N}^{h}, u_{T}^{h}\right) \geq 0$, and choosing $v_{T}^{h}=2 u_{T}^{h}$ gives $\int_{\Gamma_{C}} \lambda_{T}^{h} \cdot u_{T}^{h} d \Gamma+j\left(\mathcal{F} \lambda_{N}^{h}, u_{T}^{h}\right) \geq 0$, which implies the complementarity relation

$$
\int_{\Gamma_{C}} \lambda_{T}^{h} \cdot u_{T}^{h} d \Gamma+j\left(\mathcal{F} \lambda_{N}^{h}, u_{T}^{h}\right)=0 .
$$


Taking this into account in (2.8) leads to

$$
\int_{\Gamma_{C}} \lambda_{T}^{h} \cdot v_{T}^{h} d \Gamma+j\left(\mathcal{F} \lambda_{N}^{h}, v_{T}^{h}\right) \geq 0 \quad \forall v_{T}^{h} \in X_{T}^{h}
$$

which implies $\lambda_{T}^{h} \in \Lambda_{T}^{h}\left(\mathcal{F} \lambda_{N}^{h}\right)$. Now, from the complementarity relation and for all $\mu_{T}^{h} \in \Lambda_{T}^{h}\left(\mathcal{F} \lambda_{N}^{h}\right)$ one has

$$
\int_{\Gamma_{C}}\left(\mu_{T}^{h}-\lambda_{T}^{h}\right) \cdot u_{T}^{h} d \Gamma=\int_{\Gamma_{C}} \mu_{T}^{h} \cdot u_{T}^{h} d \Gamma+j\left(\mathcal{F} \lambda_{N}^{h}, u_{T}^{h}\right) \geq 0
$$

which implies (2.7).

Remark 3. The equivalence given by this lemma is a classical result when it deals with the continuous problem. With the particular finite element discretization considered in this section, the result is still valid in the finite-dimensional case. One of the reasons is that the space of the multipliers has been chosen in such a way that it can represent the dual space of the discrete trace space $X_{T}^{h}$. But this result does not remain valid when a smaller space for the multipliers is chosen.

\section{The error estimate.}

THEOREM 3.1. Let $(u, \lambda)$ be the solution to problem (1.8) (for $d=2$ ) such that $\lambda_{T}=\mathcal{F} \lambda_{N} \xi$, with $\xi \in M\left(X_{T} \rightarrow X_{N}\right), \xi \in D i r_{T}\left(u_{T}\right)$ a.e. on $\Gamma_{C}$, and $\mathcal{F}<$ $c_{i s}\left(C_{\alpha}\|\xi\|_{a}\right)^{-1}$. Assume that $u \in\left(H^{(3 / 2)+\varepsilon}(\Omega)\right)^{2}$ with $\varepsilon>0$, and let $\left(u^{h}, \lambda^{h}\right)$ be a solution to the discrete problem (2.3). Then there exists a constant $C>0$ independent of $h$ and $u$ such that

$$
\left\|u-u^{h}\right\|_{a}+\left\|\lambda-\lambda^{h}\right\|_{-a, \Gamma_{C}} \leq C h^{1 / 2}\|u\|_{\left(H^{(3 / 2)+\varepsilon}(\Omega)\right)^{2}} .
$$

Proof. Let $v^{h} \in V^{h}$. Then

$$
\begin{aligned}
\left\|u-u^{h}\right\|_{a}^{2}= & a\left(u-u^{h}, u-u^{h}\right) \\
= & a\left(u-u^{h}, u-v^{h}\right)+a\left(u, v^{h}-u^{h}\right)-a\left(u^{h}, v^{h}-u^{h}\right) \\
= & a\left(u-u^{h}, u-v^{h}\right)+\int_{\Gamma_{C}} \lambda_{N}\left(v_{N}^{h}-u_{N}^{h}\right) d \Gamma+\int_{\Gamma_{C}} \lambda_{T} \cdot\left(v_{T}^{h}-u_{T}^{h}\right) d \Gamma \\
& -\int_{\Gamma_{C}} \lambda_{N}^{h}\left(v_{N}^{h}-u_{N}^{h}\right) d \Gamma-\int_{\Gamma_{C}} \lambda_{T}^{h} \cdot\left(v_{T}^{h}-u_{T}^{h}\right) d \Gamma \\
= & a\left(u-u^{h}, u-v^{h}\right) \\
& +\int_{\Gamma_{C}}\left(\lambda_{N}-\lambda_{N}^{h}\right)\left(v_{N}^{h}-u_{N}\right) d \Gamma+\int_{\Gamma_{C}}\left(\lambda_{T}-\lambda_{T}^{h}\right) \cdot\left(v_{T}^{h}-u_{T}\right) d \Gamma \\
& +\int_{\Gamma_{C}}\left(\lambda_{N}-\lambda_{N}^{h}\right)\left(u_{N}-u_{N}^{h}\right) d \Gamma+\int_{\Gamma_{C}}\left(\lambda_{T}-\lambda_{T}^{h}\right) \cdot\left(u_{T}-u_{T}^{h}\right) d \Gamma .
\end{aligned}
$$

The continuous and discrete complementary conditions imply

$$
\int_{\Gamma_{C}} \lambda_{N} u_{N} d \Gamma=\int_{\Gamma_{C}} \lambda_{N}^{h} u_{N}^{h} d \Gamma=0 .
$$


Hence

$$
\begin{aligned}
\left\|u-u^{h}\right\|_{a}^{2}= & a\left(u-u^{h}, u-v^{h}\right) \\
& +\int_{\Gamma_{C}}\left(\lambda_{N}-\lambda_{N}^{h}\right)\left(v_{N}^{h}-u_{N}\right) d \Gamma+\int_{\Gamma_{C}}\left(\lambda_{T}-\lambda_{T}^{h}\right) \cdot\left(v_{T}^{h}-u_{T}\right) d \Gamma \\
& -\int_{\Gamma_{C}} \lambda_{N} u_{N}^{h}+\lambda_{N}^{h} u_{N} d \Gamma+\int_{\Gamma_{C}}\left(\lambda_{T}-\lambda_{T}^{h}\right) \cdot\left(u_{T}-u_{T}^{h}\right) d \Gamma .
\end{aligned}
$$

Using the continuity of the bilinear form, we obtain

$$
\begin{aligned}
\left\|u-u^{h}\right\|_{a}^{2} \leq & \left\|u-u^{h}\right\|_{a}\left\|u-v^{h}\right\|_{a}+\left\|\lambda-\lambda^{h}\right\|_{-a, \Gamma_{C}}\left\|u-v^{h}\right\|_{a, \Gamma_{C}} \\
& -\int_{\Gamma_{C}} \lambda_{N} u_{N}^{h}+\lambda_{N}^{h} u_{N} d \Gamma+\int_{\Gamma_{C}}\left(\lambda_{T}-\lambda_{T}^{h}\right) \cdot\left(u_{T}-u_{T}^{h}\right) d \Gamma .
\end{aligned}
$$

Additionally, we consider the equilibrium equation. From $V^{h} \subset V$, we get

$$
a\left(u, v^{h}\right)=l\left(v^{h}\right)+\int_{\Gamma_{C}} \lambda \cdot v^{h} d \Gamma \quad \forall v^{h} \in V^{h} .
$$

Since

$$
a\left(u^{h}, v^{h}\right)=l\left(v^{h}\right)+\int_{\Gamma_{C}} \lambda^{h} \cdot v^{h} d \Gamma \quad \forall v^{h} \in V^{h},
$$

we deduce by subtraction that

$$
a\left(u-u^{h}, v^{h}\right)=\int_{\Gamma_{C}}\left(\lambda-\lambda^{h}\right) \cdot v^{h} d \Gamma \quad \forall v^{h} \in V^{h} .
$$

Consequently, for any $v^{h} \in V^{h}$ and any $\mu^{h} \in X^{h}$

$$
\begin{aligned}
\int_{\Gamma_{C}}\left(\lambda^{h}-\mu^{h}\right) \cdot v^{h} d \Gamma & =a\left(u^{h}-u, v^{h}\right)+\int_{\Gamma_{C}}\left(\lambda-\mu^{h}\right) \cdot v^{h} d \Gamma \\
& \leq\left(\left\|u-u^{h}\right\|_{a}+\left\|\lambda-\mu^{h}\right\|_{-a, \Gamma_{C}}\right)\left\|v^{h}\right\|_{a} .
\end{aligned}
$$

The mesh independent inf-sup condition (2.6) implies, for any $\mu^{h} \in X^{h}$,

$$
c_{i s}\left\|\lambda^{h}-\mu^{h}\right\|_{-a, \Gamma_{C}} \leq \sup _{v^{h} \in V^{h}} \frac{\int_{\Gamma_{C}}\left(\lambda^{h}-\mu^{h}\right) \cdot v^{h} d \Gamma}{\left\|v^{h}\right\|_{a}} \leq\left\|u-u^{h}\right\|_{a}+\left\|\lambda-\mu^{h}\right\|_{-a, \Gamma_{C}} .
$$

By the triangular inequality we come to the conclusion that

$$
\left\|\lambda-\lambda^{h}\right\|_{-a, \Gamma_{C}} \leq \frac{1}{c_{i s}}\left\|u-u^{h}\right\|_{a}+\left(1+\frac{1}{c_{i s}}\right) \inf _{\mu^{h} \in X^{h}}\left\|\lambda-\mu^{h}\right\|_{-a, \Gamma_{C}} .
$$

Keeping in mind that $u \in\left(H^{(3 / 2)+\varepsilon}(\Omega)\right)^{2}$ with $\varepsilon>0$, so that $\lambda \in\left(L^{2}\left(\Gamma_{C}\right)\right)^{2}$ according to the trace theorem, we choose $v^{h}=I^{h} u$, where $I^{h}$ denotes the Lagrange interpolation operator mapping onto $V^{h}$, and $\mu^{h}=\pi^{h} \lambda$, where $\pi^{h}$ represents the $\left(L^{2}\left(\Gamma_{C}\right)\right)^{2}$-projection operator mapping onto $X^{h}$. As a consequence $($ see $[7,9,13])$ if $\varepsilon>0$ is small enough, we have

$$
\inf _{v^{h} \in V^{h}}\left\|u-v^{h}\right\|_{a} \leq C h^{(1 / 2)+\varepsilon}\|u\|_{\left(H^{(3 / 2)+\varepsilon}(\Omega)\right)^{2}}
$$


and

$$
\inf _{\mu^{h} \in X^{h}}\left\|\lambda-\mu^{h}\right\|_{-a, \Gamma_{C}} \leq C h^{(1 / 2)+\varepsilon}\|u\|_{\left(H^{(3 / 2)+\varepsilon}(\Omega)\right)^{2}},
$$

where $C$ denotes here and afterwards a positive constant independent of $h$. We now estimate the terms in (3.2) coming from the contact approximation. Since $u_{N} \leq 0$ and $\lambda_{N}^{h} \leq 0$ on $\Gamma_{C}$, we deduce that the first term is nonpositive:

$$
-\int_{\Gamma_{C}} \lambda_{N}^{h} u_{N} d \Gamma \leq 0
$$

In order to estimate the second term in (3.2) coming from the contact approximation we introduce a specific operator. Namely, $r^{h}: L^{1}\left(\Gamma_{C}\right) \mapsto X_{N}^{h}$ is the quasi-interpolation operator defined for any function $v$ in $L^{1}\left(\Gamma_{C}\right)$ by

$$
r^{h} v=\sum_{x \in N^{h}} \alpha_{x}(v) \psi_{x}
$$

where $N^{h}$ represents the set of nodes of $\overline{\Gamma_{C}}, \psi_{x}$ is the scalar basis function of $X_{N}^{h}$ (defined on $\overline{\Gamma_{C}}$ ) at node $x$ verifying $\psi_{x}\left(x^{\prime}\right)=\delta_{x, x^{\prime}}$ for all $x^{\prime} \in N^{h}$, and

$$
\alpha_{x}(v)=\left(\int_{\Gamma_{C}} v \psi_{x} d \Gamma\right)\left(\int_{\Gamma_{C}} \psi_{x} d \Gamma\right)^{-1} .
$$

Remark 4. It is straightforward to check that $r^{h}$ is linear and that it preserves nonpositivity. It is also obvious that $r^{h} v^{h} \neq v^{h}$ when $v^{h} \in X_{N}^{h}$. This operator is different from Clément's (which consists of making local projections onto $P_{1}$ functions; see [10]), from Chen-Nochetto's (which uses local projections onto $P_{0}$ functions; see $[8]$ ), and from Ben Belgacem-Renard's (which consists of making local projections onto the convex cone of nonpositive $P_{1}$ functions; see [6]). The main particularity of the operator $r^{h}$, which directly follows from its definition, is that $r^{h} v^{h} \leq 0$ when $v^{h} \in X_{N}^{h}$ satisfies only "weak nonpositivity conditions"; i.e.,

$$
\int_{\Gamma_{C}} \mu_{N}^{h} v^{h} d \Gamma \geq 0 \quad \forall \mu_{N}^{h} \in \Lambda_{N}^{h}
$$

This property is not satisfied by the operators in [8] and [10]. Moreover, as we see hereafter, the approximation properties of $r^{h}$ hold for any function without sign condition, contrary to the operator in [6].

The approximation properties of $r^{h}$ are established in [28]. We recall them to render the proof of Theorem 3.1 self-contained. We first show the $L^{2}$-stability property of $r^{h}$.

LEMma 3.2. There is a positive constant $C$ independent of $h$ such that for any $v \in L^{2}\left(\Gamma_{C}\right)$ and any $E \in E_{C}^{h}\left(E_{C}^{h}\right.$ denotes the set of closed edges lying in $\left.\overline{\Gamma_{C}}\right)$

$$
\left\|r^{h} v\right\|_{L^{2}(E)} \leq C\|v\|_{L^{2}\left(\gamma_{E}\right)},
$$

where $\gamma_{E}=\cup_{\left\{F \in E_{C}^{h}: F \cap E \neq \emptyset\right\}} F$.

Proof. Let $\gamma_{x}$ be the support of the basis function $\psi_{x}$ in $\Gamma_{C}$. Using the definition of $\alpha_{x}(v)$, the Cauchy-Schwarz inequality, and the uniform regularity of the mesh, we get

$$
\left|\alpha_{x}(v)\right| \leq\|v\|_{L^{2}\left(\gamma_{x}\right)}\left\|\psi_{x}\right\|_{L^{2}\left(\gamma_{x}\right)}\left\|\psi_{x}\right\|_{L^{1}\left(\gamma_{x}\right)}^{-1} \leq C h^{-\frac{1}{2}}\|v\|_{L^{2}\left(\gamma_{x}\right)} .
$$


We obtain by a triangular inequality

$$
\left\|r^{h} v\right\|_{L^{2}(E)}=\left\|\sum_{x \in N^{h} \cap E} \alpha_{x}(v) \psi_{x}\right\|_{L^{2}(E)} \leq C\|v\|_{L^{2}\left(\gamma_{E}\right)} .
$$

The next lemma is concerned with the $L^{2}$-approximation properties of $r^{h}$.

LEMMA 3.3. There is a positive constant $C$ independent of $h$ such that for any $v \in H^{\eta}\left(\Gamma_{C}\right), 0 \leq \eta \leq 1$, and any $E \in E_{C}^{h}\left(E_{C}^{h}\right.$ denotes the set of closed edges lying in $\left.\overline{\Gamma_{C}}\right)$

$$
\left\|v-r^{h} v\right\|_{L^{2}(E)} \leq C h^{\eta}\|v\|_{H^{\eta}\left(\gamma_{E}\right)}
$$

where $\gamma_{E}=\cup_{\left\{F \in E_{C}^{h}: F \cap E \neq \emptyset\right\}} F$.

Proof. When $\eta=0$ the bound results from the previous lemma. Note that $r^{h}$ preserves the constant functions on $\Gamma_{C}$. Let be given an arbitrary constant function $c(x)=c$, for all $x \in \Gamma_{C}$. From the definition of $r^{h}$, we may write, for any $v \in H^{\eta}\left(\Gamma_{C}\right)$,

$$
v-r^{h} v=v-c-r^{h}(v-c) .
$$

Therefore by Lemma 3.2 we get

$$
\left\|v-r^{h} v\right\|_{L^{2}(E)} \leq C\left(\|v-c\|_{L^{2}(E)}+\|v-c\|_{L^{2}\left(\gamma_{E}\right)}\right) \leq C\|v-c\|_{L^{2}\left(\gamma_{E}\right)} \quad \forall c \in R .
$$

We then choose $c=\int_{\gamma_{E}} v(x) d x /\left|\gamma_{E}\right|$ in (3.9), where $\left|\gamma_{E}\right|$ denotes the length of $\gamma_{E}$. Then if $x \in \gamma_{E}$ and $0<\eta<1$, we have

$$
\begin{aligned}
v(x)-c & =\left|\gamma_{E}\right|^{-1} \int_{\gamma_{E}} v(x)-v(y) d y \\
& =\left|\gamma_{E}\right|^{-1} \int_{\gamma_{E}} \frac{v(x)-v(y)}{|x-y|^{\frac{1+2 \eta}{2}}}|x-y|^{\frac{1+2 \eta}{2}} d y .
\end{aligned}
$$

Using the Cauchy-Schwarz inequality, we deduce

$$
\begin{aligned}
\int_{\gamma_{E}}(v(x)-c)^{2} d x & =\left|\gamma_{E}\right|^{-2} \int_{\gamma_{E}}\left(\int_{\gamma_{E}} \frac{v(x)-v(y)}{|x-y|^{\frac{1+2 \eta}{2}}}|x-y|^{\frac{1+2 \eta}{2}} d y\right)^{2} d x \\
& \leq\left|\gamma_{E}\right|^{-2} \int_{\gamma_{E}}\left(\int_{\gamma_{E}} \frac{(v(x)-v(y))^{2}}{|x-y|^{1+2 \eta}} d y \int_{\gamma_{E}}|x-y|^{1+2 \eta} d y\right) d x \\
& \leq\left|\gamma_{E}\right|^{2 \eta} \int_{\gamma_{E}} \int_{\gamma_{E}} \frac{(v(x)-v(y))^{2}}{|x-y|^{1+2 \eta}} d y d x \\
& \leq C h^{2 \eta}\|v\|_{H^{\eta}\left(\gamma_{E}\right)}^{2} .
\end{aligned}
$$

Hence the result.

If $x \in \gamma_{E}$ and $\eta=1$, we have

$$
v(x)-c=\left|\gamma_{E}\right|^{-1} \int_{\gamma_{E}} v(x)-v(y) d y=\left|\gamma_{E}\right|^{-1} \int_{\gamma_{E}} \int_{y}^{x} v^{\prime}(t) d t d y
$$

Hence

$$
|v(x)-c| \leq\left|\gamma_{E}\right|^{\frac{1}{2}}\left\|v^{\prime}\right\|_{L^{2}\left(\gamma_{E}\right)}
$$


The result is then straightforward.

End of the proof of Theorem 3.1. The second term coming from the contact approximation in (3.2) is handled as follows:

$$
-\int_{\Gamma_{C}} \lambda_{N} u_{N}^{h} d \Gamma=-\int_{\Gamma_{C}} \lambda_{N}\left(u_{N}^{h}-r^{h} u_{N}^{h}\right) d \Gamma-\int_{\Gamma_{C}} \lambda_{N} r^{h} u_{N}^{h} d \Gamma .
$$

According to (2.3), $u_{N}^{h}$ satisfies a weak nonnegativity condition as in (3.7). From Remark 4 we deduce that $r^{h} u_{N}^{h} \leq 0$. Hence we have, for any small $\varepsilon>0$,

$$
\begin{aligned}
-\int_{\Gamma_{C}} \lambda_{N} u_{N}^{h} d \Gamma \leq & -\int_{\Gamma_{C}} \lambda_{N}\left(u_{N}^{h}-r^{h} u_{N}^{h}\right) d \Gamma \\
\leq & \left\|\lambda_{N}\right\|_{L^{2}\left(\Gamma_{C}\right)}\left\|u_{N}^{h}-r^{h} u_{N}^{h}\right\|_{L^{2}\left(\Gamma_{C}\right)} \\
\leq & \left\|\lambda_{N}\right\|_{L^{2}\left(\Gamma_{C}\right)}\left\|\left(u_{N}^{h}-u_{N}\right)-r^{h}\left(u_{N}^{h}-u_{N}\right)\right\|_{L^{2}\left(\Gamma_{C}\right)} \\
& +\left\|\lambda_{N}\right\|_{L^{2}\left(\Gamma_{C}\right)}\left\|u_{N}-r^{h} u_{N}\right\|_{L^{2}\left(\Gamma_{C}\right)} \\
\leq & C h^{1 / 2}\|u\|_{\left(H^{(3 / 2)+\varepsilon}(\Omega)\right)^{2}}\left\|u_{N}^{h}-u_{N}\right\|_{H^{1 / 2}\left(\Gamma_{C}\right)} \\
& +C h\|u\|_{\left(H^{(3 / 2)+\varepsilon}(\Omega)\right)^{2}}\left\|u_{N}\right\|_{H^{1}\left(\Gamma_{C}\right)},
\end{aligned}
$$

where the trace theorem $\left\|\lambda_{N}\right\|_{L^{2}\left(\Gamma_{C}\right)} \leq C\|u\|_{\left(H^{(3 / 2)+\varepsilon}(\Omega)\right)^{2}}$ (see [20]) and the estimates in Lemma 3.3 have been used. Putting together estimates (3.6) and (3.10) yields for any small $\varepsilon>0$

$$
\begin{aligned}
& -\int_{\Gamma_{C}} \lambda_{N}^{h} u_{N}+\lambda_{N} u_{N}^{h} d \Gamma \leq C h^{1 / 2}\|u\|_{\left(H^{(3 / 2)+\varepsilon}(\Omega)\right)^{2}} \\
& \left(\left\|u-u^{h}\right\|_{a}+h^{1 / 2}\|u\|_{\left(H^{(3 / 2)+\varepsilon}(\Omega)\right)^{2}}\right) .
\end{aligned}
$$

We now estimate the terms corresponding to the friction approximation in (3.2). From the assumptions in the theorem we write

$$
\begin{aligned}
\int_{\Gamma_{C}}\left(\lambda_{T}-\lambda_{T}^{h}\right) \cdot\left(u_{T}-u_{T}^{h}\right) d \Gamma & =\int_{\Gamma_{C}}\left(\lambda_{T}-\mathcal{F} \lambda_{N}^{h} \xi\right) \cdot\left(u_{T}-u_{T}^{h}\right) d \Gamma \\
& +\int_{\Gamma_{C}}\left(\mathcal{F} \lambda_{N}^{h} \xi-\lambda_{T}^{h}\right) \cdot\left(u_{T}-u_{T}^{h}\right) d \Gamma \\
& =\int_{\Gamma_{C}} \mathcal{F}\left(\lambda_{N}-\lambda_{N}^{h}\right) \xi \cdot\left(u_{T}-u_{T}^{h}\right) d \Gamma \\
& +\int_{\Gamma_{C}}\left(\mathcal{F} \lambda_{N}^{h} \xi-\lambda_{T}^{h}\right) \cdot\left(u_{T}-u_{T}^{h}\right) d \Gamma
\end{aligned}
$$

The estimate of the first integral term in (3.12) gives

$$
\int_{\Gamma_{C}} \mathcal{F}\left(\lambda_{N}-\lambda_{N}^{h}\right) \xi \cdot\left(u_{T}-u_{T}^{h}\right) d \Gamma \leq C_{\alpha} \mathcal{F}\|\xi\|_{a}\left\|\lambda-\lambda^{h}\right\|_{-a, \Gamma_{C}}\left\|u-u^{h}\right\|_{a}
$$

The second integral term in (3.12) is written as follows:

$$
\begin{aligned}
\int_{\Gamma_{C}}\left(\mathcal{F} \lambda_{N}^{h} \xi-\lambda_{T}^{h}\right) \cdot\left(u_{T}-u_{T}^{h}\right) d \Gamma= & \int_{\Gamma_{C}} \mathcal{F} \lambda_{N}^{h} \xi \cdot\left(u_{T}-u_{T}^{h}\right) d \Gamma-\int_{\Gamma_{C}} \lambda_{T}^{h} \cdot u_{T} d \Gamma \\
& +\int_{\Gamma_{C}} \lambda_{T}^{h} \cdot u_{T}^{h} d \Gamma .
\end{aligned}
$$


Using the equivalent discrete friction conditions in (2.8), we obtain for any $v^{h} \in V^{h}$

$$
\begin{aligned}
\int_{\Gamma_{C}}\left(\mathcal{F} \lambda_{N}^{h} \xi-\lambda_{T}^{h}\right) \cdot\left(u_{T}-u_{T}^{h}\right) d \Gamma & \leq \int_{\Gamma_{C}} \mathcal{F} \lambda_{N}^{h} \xi \cdot\left(u_{T}-u_{T}^{h}\right) d \Gamma-\int_{\Gamma_{C}} \lambda_{T}^{h} \cdot u_{T} d \Gamma \\
& +\int_{\Gamma_{C}} \lambda_{T}^{h} \cdot v_{T}^{h} d \Gamma-\int_{\Gamma_{C}} \mathcal{F} \lambda_{N}^{h}\left|v_{T}^{h}\right| d \Gamma+\int_{\Gamma_{C}} \mathcal{F} \lambda_{N}^{h}\left|u_{T}^{h}\right| d \Gamma .
\end{aligned}
$$

Choosing $v_{T}^{h}=I^{h} u_{T}$ and since $\xi \cdot u_{T}=\left|u_{T}\right|$, we obtain

$$
\begin{aligned}
\int_{\Gamma_{C}}\left(\mathcal{F} \lambda_{N}^{h} \xi-\lambda_{T}^{h}\right) \cdot\left(u_{T}-u_{T}^{h}\right) d \Gamma \leq & \int_{\Gamma_{C}} \lambda_{T}^{h} \cdot\left(I^{h} u_{T}-u_{T}\right) d \Gamma+\int_{\Gamma_{C}} \mathcal{F} \lambda_{N}^{h}\left(\left|u_{T}^{h}\right|-\xi \cdot u_{T}^{h}\right) d \Gamma \\
& +\int_{\Gamma_{C}} \mathcal{F} \lambda_{N}^{h}\left(\left|u_{T}\right|-\left|I^{h} u_{T}\right|\right) d \Gamma .
\end{aligned}
$$

The estimate of the first term in (3.13) is achieved as follows by using the error estimates in [9]:

$$
\begin{aligned}
\int_{\Gamma_{C}} \lambda_{T}^{h} \cdot\left(I^{h} u_{T}-u_{T}\right) d \Gamma & =\int_{\Gamma_{C}}\left(\lambda_{T}^{h}-\lambda_{T}\right) \cdot\left(I^{h} u_{T}-u_{T}\right) d \Gamma+\int_{\Gamma_{C}} \lambda_{T} \cdot\left(I^{h} u_{T}-u_{T}\right) d \Gamma \\
& \leq C h^{1 / 2}\|u\|_{\left(H^{3 / 2}(\Omega)\right)^{2}}\left(\left\|\lambda-\lambda^{h}\right\|_{-a, \Gamma_{C}}+h^{1 / 2}\|u\|_{\left(H^{(3 / 2)+\varepsilon}(\Omega)\right)^{2}}\right) .
\end{aligned}
$$

The estimate of the second term in (3.13) uses the fact that $\lambda_{N}^{h} \leq 0$ and $\left|u_{T}^{h}\right|-$ $\xi . u_{T}^{h} \geq 0$ so that

$$
\int_{\Gamma_{C}} \mathcal{F} \lambda_{N}^{h}\left(\left|u_{T}^{h}\right|-\xi \cdot u_{T}^{h}\right) d \Gamma \leq 0 .
$$

Finally, the third term in (3.13) yields

$$
\begin{aligned}
\int_{\Gamma_{C}} \mathcal{F} \lambda_{N}^{h}\left(\left|u_{T}\right|-\left|I^{h} u_{T}\right|\right) d \Gamma & \leq \mathcal{F}\left\|\lambda_{N}^{h}\right\|_{L^{2}\left(\Gamma_{C}\right)}\left\|\left|u_{T}\right|-\left|I^{h} u_{T}\right|\right\|_{L^{2}\left(\Gamma_{C}\right)} \\
& \leq \mathcal{F}\left\|\lambda_{N}^{h}\right\|_{L^{2}\left(\Gamma_{C}\right)}\left\|u_{T}-I^{h} u_{T}\right\|_{L^{2}\left(\Gamma_{C}\right)} \\
& \leq C \mathcal{F}\left\|\lambda_{N}^{h}\right\|_{L^{2}\left(\Gamma_{C}\right)} h\|u\|_{\left(H^{3 / 2}(\Omega)\right)^{2}}
\end{aligned}
$$

Further, using the (global) $L^{2}\left(\Gamma_{C}\right)$-projection operator $\pi_{N}^{h}$ onto $X_{N}^{h}$ (the notation $\pi^{h}$ stands for the $\left(L^{2}\left(\Gamma_{C}\right)\right)^{2}$-projection operator onto $\left.X^{h}\right)$ and an inverse inequality (see, e.g., $[7,9,13])$, we write

$$
\begin{aligned}
\left\|\lambda_{N}^{h}\right\|_{L^{2}\left(\Gamma_{C}\right)} & \leq\left\|\lambda_{N}^{h}-\pi_{N}^{h} \lambda_{N}\right\|_{L^{2}\left(\Gamma_{C}\right)}+\left\|\pi_{N}^{h} \lambda_{N}-\lambda_{N}\right\|_{L^{2}\left(\Gamma_{C}\right)}+\left\|\lambda_{N}\right\|_{L^{2}\left(\Gamma_{C}\right)} \\
& \leq C\left(h^{-1 / 2}\left\|\lambda^{h}-\pi^{h} \lambda\right\|_{-a, \Gamma_{C}}+\left\|\lambda_{N}\right\|_{L^{2}\left(\Gamma_{C}\right)}\right) \\
& \leq C\left(h^{-1 / 2}\left\|\lambda-\lambda^{h}\right\|_{-a, \Gamma_{C}}+\|u\|_{\left(H^{(3 / 2)+\varepsilon}(\Omega)\right)^{2}}\right) .
\end{aligned}
$$

Therefore,

$$
\begin{gathered}
\int_{\Gamma_{C}} \mathcal{F} \lambda_{N}^{h}\left(\left|u_{T}\right|-\left|I^{h} u_{T}\right|\right) d \Gamma \leq C \mathcal{F} h^{1 / 2}\|u\|_{\left(H^{3 / 2}(\Omega)\right)^{2}} \\
\left(\left\|\lambda-\lambda^{h}\right\|_{-a, \Gamma_{C}}+h^{1 / 2}\|u\|_{\left(H^{(3 / 2)+\varepsilon}(\Omega)\right)^{2}}\right) .
\end{gathered}
$$


We come to the conclusion that the term dealing with the friction approximation in (3.2) is bounded as follows:

$$
\begin{aligned}
\int_{\Gamma_{C}}\left(\lambda_{T}-\lambda_{T}^{h}\right) \cdot\left(u_{T}-u_{T}^{h}\right) d \Gamma \leq & C_{\alpha} \mathcal{F}\|\xi\|_{a}\left\|\lambda-\lambda^{h}\right\|_{-a, \Gamma_{C}}\left\|u-u^{h}\right\|_{a} \\
& +C(1+\mathcal{F}) h\|u\|_{\left(H^{(3 / 2)+\varepsilon}(\Omega)\right)^{2}}^{2} \\
& +C(1+\mathcal{F}) h^{1 / 2}\|u\|_{\left(H^{3 / 2}(\Omega)\right)^{2}}\left\|\lambda-\lambda^{h}\right\|_{-a, \Gamma_{C}} .
\end{aligned}
$$

Finally, the result is obtained by using (3.4)-(3.5) and putting together (3.2), (3.3), (3.11), and (3.14).

Remark 5. The quasi-optimal rate of convergence of order $1 / 2$ in the theorem does not depend on $\varepsilon>0$. Actually we are not able to obtain a better convergence rate even if $\varepsilon$ increases. A bit more regularity than $H^{3 / 2}$ is needed to apply the trace theorem, when writing $\left\|\lambda_{N}\right\|_{L^{2}\left(\Gamma_{C}\right)} \leq C\|u\|_{\left(H^{(3 / 2)+\varepsilon}(\Omega)\right)^{2}}$ (see [20]). The choice of the regularity assumptions $u \in\left(H^{(3 / 2)+\varepsilon}(\Omega)\right)^{2}$ in the numerical analysis of contact problems is discussed in [4, Remark 2.4(i)] and [5, Remark 4.4]. If $u$ is less regular than $H^{3 / 2}$, then the normal and tangential constraints cannot be expressed pointwise, and the frictional contact conditions cannot be simply written as in (1.5)-(1.7). In the frictionless case, when $u \in\left(H^{\nu}(\Omega)\right)^{2}$ with $1<\nu<3 / 2$, the error analysis of a finite element approximation is achieved in [4]. Actually we are not able to extend these results to the frictional case.

Remark 6. If one (or both) end points of $\overline{\Gamma_{C}}=\left[x_{0}, x_{n}\right]$ is subjected to Dirichlet conditions, then the previous study can be extended with some modifications. Suppose, for instance, that $\overline{\Gamma_{C}} \cap \overline{\Gamma_{D}}=\left\{x_{0}\right\}$ and that the definition of $V^{h}$ in (2.1) remains unchanged. If we still keep the same definition of $X^{h}$ as in (2.2), then the estimate (3.5), does not hold in the general case. Thus we use a mortar approach introduced in [7]: denoting by $x_{i}, 0 \leq i \leq n$, the nodes on $\overline{\Gamma_{C}}$, we set

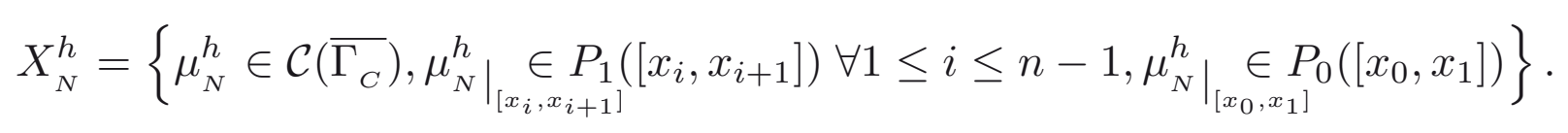

The particularity of this space is that the functions are constant on the extreme segment $\left[x_{0}, x_{1}\right]$. We choose the same kind of approximation for $X_{T}^{h}$, and we set $X^{h}=X_{N}^{h} \times X_{T}^{h}$. In this case the discrete Babuška-Brezzi inf-sup condition (2.6) still holds (see [3]). Moreover, estimate (3.5) remains valid (see [7, Lemma 4.1]). Keeping the same definitions of $\Lambda_{N}^{h}$ and $\Lambda_{T}^{h}\left(\mathcal{F} \lambda_{N}^{h}\right)$ as in (2.4) and (2.5), we note that the equivalence in Lemma 2.1 still holds in this case since the dimensions of the multiplier and tangential displacement spaces are the same (see also Remark 1) and the inf-sup condition is satisfied. According to [30], problem (2.3) admits a solution for any friction coefficient, and the solution is unique for a sufficiently small friction coefficient. The following result is then obtained: if $\left(u^{h}, \lambda^{h}\right)$ is a solution to (2.3), then estimate (3.1) is recovered.

Remark 7. In the three-dimensional case, the convergence result should hold (at least when $\overline{\Gamma_{C}} \cap \overline{\Gamma_{D}}=\emptyset$ ), and the main task would be to generalize the estimate (3.8).

Remark 8. If $\mathcal{F}=0$, then the continuous problem admits a unique solution. Choosing then the same approximation method as in (2.3) (therefore $\lambda_{T}^{h}=0$ and the discrete solution is unique) and accomplishing the convergence analysis has led to an upper bound of the error of order $h^{1 / 2}$ under $H^{2}$-regularity hypotheses (see [6]). The estimate obtained in the present paper improves the bound in [6], since we obtain the 
same convergence rate with fewer regularity assumptions $\left(H^{(3 / 2)+\varepsilon}\right.$ with $\varepsilon$ arbitrary small instead of $H^{2}$ ). Moreover, we observe that there is no loss of convergence when the friction terms are added. Nevertheless we mention that there exists in the frictionless case a standard finite element approximation, which leads to an upper bound of the error of order $h^{3 / 4}$ under $H^{2}$-regularity hypotheses (see [24, 23]) and of order $h$ with some additional assumptions concerning the finiteness of transition points between contact and separation (see [29]). Actually we are not able to extend these results to the frictional case.

Remark 9. Note that we do not prove that the solution to the discrete problem is unique under the assumptions of Theorem 3.1. This seems to be an open question which is actually under investigation. Note also that this possible loss of uniqueness would not be embarrassing in the a priori error analysis of Theorem 3.1. As a matter of fact, even if there are multiple solutions to the discrete problem, any solution would converge towards the unique solution of the continuous model. Additionally, the bound ensuring uniqueness in Proposition 1.2 is $\mathcal{F}<\left(C_{\alpha}\|\xi\|_{a}\right)^{-1}$, and we establish the error estimate only for $\mathcal{F}<c_{i s}\left(C_{\alpha}\|\xi\|_{a}\right)^{-1}$. It should be interesting to see whether or not it is possible to prove an error estimate for all the uniqueness cases of Proposition 1.2 .

Conclusion. This work is a contribution to the numerical analysis of the unilateral contact problem governed by Coulomb's law of friction in elastostatics. As far as we know, this study establishes a first error estimate with a convergence rate for this model. From the previous remarks we can reasonably conclude that the present convergence analysis could be generalized in many directions.

\section{REFERENCES}

[1] R.A. Adams, Sobolev Spaces, Academic Press, New York, 1975.

[2] L.-E. ANDERSSON, Existence results for quasistatic contact problems with Coulomb friction, Appl. Math. Optim., 42 (2000), pp. 169-202.

[3] F. Ben Belgacem, The mortar finite element method with Lagrange multipliers, Numer. Math., 84 (1999), pp. 173-197.

[4] F. Ben Belgacem, Numerical simulation of some variational inequalities arisen from unilateral contact problems by the finite element methods, SIAM J. Numer. Anal., 37 (2000), pp. $1198-1216$.

[5] F. Ben Belgacem, P. Hild, and P. Laborde, Extension of the mortar finite element method to a variational inequality modeling unilateral contact, Math. Models Methods Appl. Sci., 9 (1999), pp. 287-303.

[6] F. Ben Belgacem and Y. Renard, Hybrid finite element methods for the Signorini problem, Math. Comp., 72 (2003), pp. 1117-1145.

[7] C. Bernardi, Y. Maday, and A.T. Patera, A new nonconforming approach to domain decomposition: The mortar element method, in Collège de France seminar, H. Brezis and J.-L. Lions, eds., Pitman, Boston, 1994, pp. 13-51.

[8] Z. Chen And R.H. Nochetto, Residual type a posteriori error estimates for elliptic obstacle problems, Numer. Math., 84 (2000), pp. 527-548.

[9] P.G. Ciarlet, The finite element method for elliptic problems, in Handbook of Numerical Analysis, Volume II, Part 1, P.G. Ciarlet and J.L. Lions, eds., North-Holland, Amsterdam, 1991, pp. 17-352.

[10] P. ClÉment, Approximation by finite elements functions using local regularization, RAIRO Anal. Numer., 9 (1975), pp. 77-84.

[11] M. Cocu, Existence of solutions of Signorini problems with friction, Internat. J. Engrg. Sci., 22 (1984), pp. 567-575.

[12] P. Coorevits, P. Hild, K. Lhalouani, and T. Sassi, Mixed finite element methods for unilateral problems: Convergence analysis and numerical studies, Math. Comp., 71 (2002), pp. $1-25$.

[13] M. Crouzeix And V. Thomée, The stability in $L^{p}$ and $W^{1, p}$ of the $L^{2}$ projection on finite element function spaces, Math. Comp., 48 (1987), pp. 521-532. 
[14] L. Demkovicz And J.T. Oden, On some existence and uniqueness results in contact problems with nonlocal friction, Nonlinear Anal., 6 (1982), pp. 1075-1093.

[15] G. Duvaut, Problèmes unilatéraux en mécanique des milieux continus, in Actes du Congrès International des Mathématiciens (Nice 1970), Tome 3, Gauthier-Villars, Paris, 1971, pp. $71-77$.

[16] G. Duvaut, Equilibre d'un solide élastique avec contact unilatéral et frottement de Coulomb, C. R. Acad. Sci. Sér. I Math., 290 (1980), pp. 263-265.

[17] G. Duvaut and J.L. Lions, Les inéquations en mécanique et en physique, Dunod, Paris, 1972.

[18] C. ECK AND J. JARUŠEK, Existence results for the static contact problem with Coulomb friction, Math. Models Methods Appl. Sci., 8 (1998), pp. 445-468.

[19] C. Eck, J. JarušEK, And M. KRBec, Unilateral Contact Problems: Variational Methods and Existence Theorems, Pure Appl. Math. 270, CRC Press, Boca Raton, FL, 2005.

[20] P. Grisvard, Elliptic Problems in Nonsmooth Domains, Pitman, Boston, 1985.

[21] W. Han and M. Sofonea, Quasistatic Contact Problems in Viscoelasticity and Viscoplasticity, American Mathematical Society, Providence, RI, 2002.

[22] J. Haslinger, Approximation of the Signorini problem with friction, Obeying the Coulomb law, Math. Methods Appl. Sci., 5 (1983), pp. 422-437.

[23] J. Haslinger and I. HlavÁČEK, Contact between elastic bodies-2. Finite element analysis, Aplikace Matematiky, 26 (1981), pp. 263-290.

[24] J. Haslinger, I. HlaváČEK, AND J. NeČAs, Numerical methods for unilateral problems in solid mechanics, in Handbook of Numerical Analysis, Volume IV, Part 2, P.G. Ciarlet and J. L. Lions, eds., North-Holland, Amsterdam, 1996, pp. 313-485.

[25] R. Hassani, P. Hild, I. Ionescu, And N.-D. Sakki, A mixed finite element method and solution multiplicity for Coulomb frictional contact, Comput. Methods Appl. Mech. Engrg., 192 (2003), pp. 4517-4531.

[26] P. HILd, Non-unique slipping in the Coulomb friction model in two-dimensional linear elasticity, Quart. J. Mech. Appl. Math., 57 (2004), pp. 225-235.

[27] P. HILD, Multiple solutions of stick and separation type in the Signorini model with Coulomb friction, Z. Angew. Math. Mech., 85 (2005), pp. 673-680.

[28] P. Hild, A Priori Error Analysis of a Sign Preserving Mixed Finite Element Method for Contact Problems, Internal Report 2006-33 of the Laboratoire de Mathématiques de Besançon; Appl. Numer. Math., submitted.

[29] S. HÜEBER AND B. I., Wohlmuth, An optimal a priori error estimate for nonlinear multibody contact problems, SIAM J. Numer. Anal., 43 (2005), pp. 156-173.

[30] H. Khenous, J. Pommier, and Y. Renard, Hybrid discretization of the Signorini problem with Coulomb friction, Theoretical aspects and comparison of some numerical solvers, Appl. Numer. Math., 56 (2006), pp. 163-192.

[31] N. Kikuchi And J.T. Oden, Contact Problems in Elasticity: A Study of Variational Inequalities and Finite Element Methods, SIAM Stud. Appl. Math. 8, SIAM, Philadelphia, 1988.

[32] A. Klarbring, A. Mikelíc, and M. Shillor, Frictional contact problems with normal compliance, Internat. J. Engrg. Sci., 26 (1988), pp. 811-832.

[33] A. Klarbring, A. Mikelíc, And M. Shillor, On friction problems with normal compliance, Nonlinear Anal., 13 (1989), pp. 935-955.

[34] P. LABORDE AND Y. RENARD, Fixed points strategies for elastostatic frictional contact problems, Math. Methods Appl. Sci., to appear.

[35] T. Laursen, Computational Contact and Impact Mechanics, Springer-Verlag, New York, 2002.

[36] J.-L. Lions and E. Magenes, Problèmes aux limites non homogènes, Dunod, Paris, 1968.

[37] J.A.C. Martins And J.T. Oden, Existence and uniqueness results for dynamic contact problems with nonlinear normal and friction interface laws, Nonlinear Anal., 11 (1987), pp. 407-428.

[38] V.G. MaZ'ya and T.O. Shaposhnikova, Theory of Multipliers in Spaces of Differentiable Functions, Pitman, Boston, 1985.

[39] J. NeČAs, J. JARUŠEK, AND J. HASLINGER, On the solution of the variational inequality to the Signorini problem with small friction, Boll. Unione Mat. Ital., 17 (1980), pp. 796-811.

[40] J.T. Oden AND J.A.C. MARTins, Models and computational methods for dynamic friction phenomena, Comput. Methods. Appl. Mech. Engrg., 52 (1985), pp. 527-634.

[41] Y. RENARD, A uniqueness criterion for the Signorini problem with Coulomb friction, SIAM J. Math. Anal., 38 (2006), pp. 452-467.

[42] R. Rocca AND M. Cocou, Numerical analysis of quasi-static unilateral contact problems with local friction, SIAM J. Numer. Anal., 39 (2001), pp. 1324-1342.

[43] P. Wriggers, Computational Contact Mechanics, Wiley, New York, 2002. 